\title{
Evolution of BL Lac host galaxies
}

\author{
J. Heidt ${ }^{1}$, M. Tröller ${ }^{1,2}$, K. Nilsson ${ }^{3}$, K. Jäger ${ }^{4}$, L. Takalo ${ }^{3}$, R. Rekola ${ }^{3}$ \\ and A. Sillanpää ${ }^{3}$ \\ ${ }^{1}$ Landessternwarte Heidelberg, Königstuhl, 69117 Heidelberg, Germany \\ ${ }^{2}$ Metsähovi Radio Observatory, Metsähovintie 114, 02540 Kylmälä, Finland \\ ${ }^{3}$ Tuorla Observatory, 21500 Piikkiö, Finland \\ ${ }^{4}$ Universitätssternwarte Göttingen, Geismarlandstr. 11, 37083 Göttingen, Germany
}

\begin{abstract}
Deep, high-resolution I-band images of 24 BL Lac objects between $\mathrm{z}=0.3$ and 1.3 taken with the NOT and the ESO-NTT and VLT telescopes are discussed. In 17/24 (71\%) of the BL Lac objects, we detected an underlying nebulosity, in 11/17 the host of the BL Lac in the redshift range $\mathrm{z}=0.3-1(<\mathrm{z}>=0.6)$. The host galaxies are luminous $\left(M_{I}=\right.$ $-25.2 \pm 0.8)$ and large $\left(r_{e}=10.5 \pm 7 \mathrm{kpc}\right)$. They are on average about 0.6 mag brighter than BL Lac host galaxies at $\mathrm{z} \sim 0.3$ indicative of evolution, whereas their half-light radii are similar. By combining our data with literature data at low-redshift and applying evolutionary models to them, we find that the properties of the host galaxies of BL Lac objects up to $\mathrm{z} \sim 1$ are compatible with passively evolving elliptical galaxies formed at a redshift of $\mathrm{z} \geqslant 2$. Host galaxies of low-luminosity radio-loud AGN (BL Lac/FR I) have very similar properties to the hosts of radio-quiet QSOs and high-luminosity radio-loud AGN (radio-loud QSO/FR II) over a wide redshift range. This supports the picture of the "Grand Unification", in which AGN activity is a transient phenomenon in galaxy evolution.
\end{abstract}

\section{Introduction}

While there is common agreement that the host galaxies of radio-quiet QSOs, radioloud QSOs and of FR II radio galaxies are practically indistinguishable from normal massive bulge-dominated galaxies with formation epochs of $z \geqslant 2-4$ followed by passive evolution thereafter (e.g. Falomo et al. 2004 and refs therein) this has not yet been illustrated for FR I radio galaxies and BL Lac objects. Since FR I radio galaxies are hard to detect at redshifts larger than $\mathrm{z}=0.2$ (e.g. Morganti et al. 1995), any cosmological evolution of the hosts of this class of AGN has to be derived from BL Lac objects. However, while the properties of BL Lac hosts at low redshift $(\mathrm{z}<0.4)$ are well known (large and luminous elliptical galaxies with $M_{R}=-23.9, r_{e}=10 \mathrm{kpc}$ similar to the RQQ and RLQ hosts, same Kormendy relation; e.g. Falomo \& Kotilainen 1999; Urry et al. 2000; Nilsson et al. 2003) observational evidence at higher redshift is poor. This is mainly due to the lack of suitable samples (only a few dozen $\mathrm{z}>0.5$ BL Lacs are known).

To overcome this problem, we observed a set of 24 BL Lac objects between $z=0.3-1.3$ $<\mathrm{z}\rangle=0.6$ very deep under mostly subarcsecond seeing conditions in the I-filter using the NOT and the ESO-NTT and VLT telescopes, the basic results of which are presented here. In the following, $\mathrm{H}_{0}=50 \mathrm{~km} \mathrm{~s}^{-1} \mathrm{Mpc}^{-1}$ and $\mathrm{q}_{0}=0$ is assumed.

\section{Results and discussion}

To analyze our images of the BL Lac objects, we fitted 3 different 2-dimensional models (AGN, AGN + bulge, AGN + disk) to the observed image via an iterative $\chi^{2}$ minimization using the Levenberg-Marquardt technique as described in Nilsson et al. 

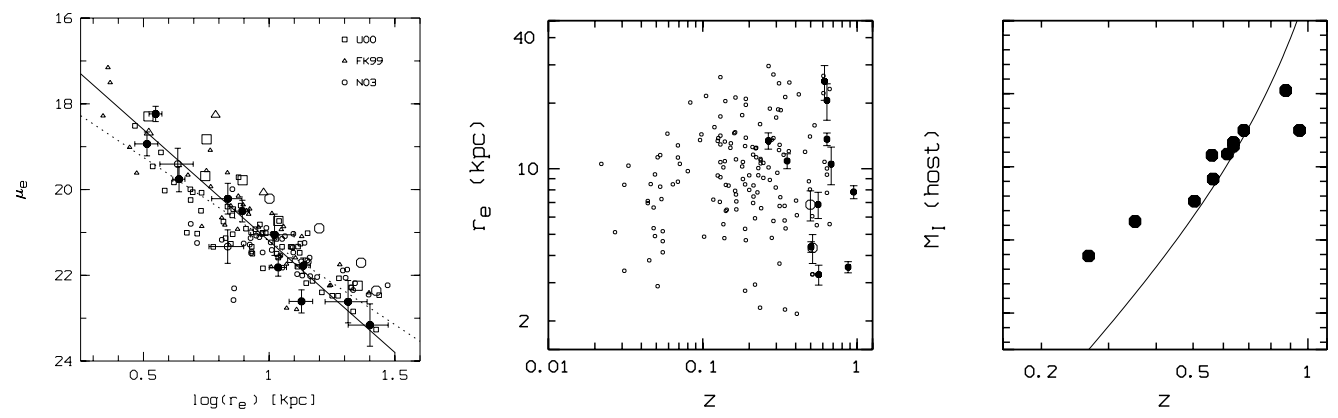

Figure 1. Left) Kormendy relation of our 11 objects (black dots) compared to observations of Falomo \& Kotilainen 1999, Urry et al. 2000 and Nilsson et al. 2003. All data have been homogeneously corrected for extinction, cosmological dimming, K-correction and evolution and converted to the R-filter. Center) Host galaxy half-light radius versus redshift from our measurements (black boxes), compared to the low-z samples of Falomo \& Kotilainen 1999, Urry et al. 2000 and Nilsson et al. 2003. The measured values are roughly constant from $\mathrm{z}=0.1 \mathrm{up}$ to $\mathrm{z}=1$. Right) Absolute host galaxy I magnitudes of our objects versus redshift. The line gives the absolute magnitude of an object with $\mathrm{I}=19$, with K-correction, but no evolution. The luminosity bias is apparent.

(2003). In 17/24 (71\%) of the BL Lac objects, we detected an underlying nebulosity. 6 of them are either intervening galaxies or are probably misidentified BL Lac objects. The remaining 11 objects span the redshift range $\mathrm{z}=0.3-1(<\mathrm{z}>=0.6)$ and have luminous $\left(M_{I}=-25.2 \pm 0.8\right)$ and large $\left(r_{e}=10.5 \pm 7 \mathrm{kpc}\right)$ elliptical host galaxies. They are on average about 0.6 mag brighter than BL Lac host galaxies at $\mathrm{z} \sim 0.3$ indicative of evolution. If we apply evolutionary models of Maraston (1988) and Maraston \& Thomas (2000) to the data (formation of the elliptical galaxy at $z \geqslant 2$ with passive evolution thereafter), our host galaxies have now $\left\langle M_{R}\right\rangle=23.9 \pm 0.4$ in perfect agreement with the results obtained for low-z samples.

In figure 1(right, center) we show the Kormendy relation and the half-light radii of our objects compared to measurements of low-z BL Lac hosts. While our high-z objects nicely occupy the same region as the low-z ones, there is basically no evolution of their half-light radii as has been found for the hosts of other types of luminous AGN. Unfortunately, our observations are affected by an luminosity bias (figure 1 left) and need to be confirmed. If they could be confirmed, this would support the picture of the "Grand Unification", in which AGN activity is a transient phenomenon in galaxy evolution.

\section{Acknowledgements}

This work was supported by the Deutsche Forschungsgemeinschaft (SFB 375, SFB 439), the VW foundation and the German Federal Ministry of Science and Technology with ID-Nos. 05 2HD50A, 05 2GO20A and 05 2MU104. J.H. acknowledges a travel grant from SFB 439 to attend this conference.

\section{References}

Falomo, R., \& Kotilainen, J. 1999, A\&A, 352, 85

Falomo, R., et al. 2004, ApJ, in press (astro-ph/0312234)

Morganti, R., et al. 1995, PASA, 12, 3

Nilsson, K., et al. 2003, A\&A, 400, 95

Urry, C. M., et al. 2000, ApJ, 532, 816 